Hepatology, Imperial College London, London, UK; ${ }^{2}$ Department of Gastroenterology, Imperial College London, London, UK; ${ }^{3}$ Centre of Pharmacology and Therapeutics, Imperial College London, London, UK; ${ }^{4}$ Division of Cancer Medicine, Surgery and Transplantation, Oslo University Hospital, Rikshospitalet, Oslo, Norway; ${ }^{5}$ UCL Institute of Hepatology, University College London, London, UK

Introduction We have previously demonstrated that the level of neutrophil gelatinase-associated lipocalin (NGAL) is increased in the bile of patients with pancreatobiliary malignancy. NGAL is expressed by activated neutrophils and many other cell types and is thought to have bacteriostatic, pro-proliferative and pro-metastatic functions. NGAL can be detected in the blood plasma. We hypothesised that the plasma NGAL level is elevated in patients with cholangiocarcinoma (CC) compared with patients with primary sclerosing cholangitis (PSC), and with healthy volunteers.

Methods Plasma samples were collected from 97 patients with confirmed CC, 62 patients with PSC and no CC and 82 healthy controls. Plasma NGAL quantification was performed in duplicate on plasma from each subject using a Quantikine ELISA kit (R\&D Systems, Minneapolis, Minnesota, USA). CC and healthy control cohorts were compared using the Student t test and receiver operator characteristic (ROC) analysis. Differences between CC and PSC cohorts were then sought. Pearson's correlation analysis was used to assess relationships between the levels of NGAL and other plasma markers.

Results Median NGAL concentrations (range) in CC, PSC and healthy controls were $92 \mathrm{ng} / \mathrm{ml}$ (14-644), 83 (43-171) and 64 (29-132) respectively. NGAL levels were significantly higher in plasma from CC patients compared with healthy controls $(p<0.0001)$. The area under the ROC curve was 0.71 (95\% CI 0.64 to $0.79 \mathrm{p}<0.0001)$. NGAL levels were significantly higher in plasma samples from the CC cohort than those from the PSC cohort $(p<0.01)$ with a ROC-AUC of 0.57 (95\% CI 0.48 to $0.65 p<0.167)$. There was no relationship between NGAL levels and CRP $\left(r^{2}=0.14\right)$, white cell count $\left(r^{2}=0.09\right)$, bilirubin $\left(r^{2}=0.01\right)$, ALP $\left(r^{2}=0.02\right)$ or creatinine $\left(r^{2}=0.03\right)$. There was moderate correlation between NGAL and Ca19-9 concentrations $\left(r^{2}=0.38\right)$.

Conclusion NGAL is expressed at significantly higher concentrations in the plasma of patients with CC compared to plasma from healthy controls and from subjects with PSC. This finding appears to be independent of renal impairment, cholestasis or systemic inflammatory response, suggesting that NGAL may represent a novel plasma biomarker of CC.

Competing interests None declared.

\section{PWE-134 SINGLE INCISION VS CONVENTIONAL LAPAROSCOPIC CHOLECYSTECTOMY: A META-ANALYSIS AND SYSTEMATIC REVIEW OF RANDOMISED CONTROLLED TRIALS}

doi:10.1136/gutjnl-2012-302514d.134

M Sajid, N Ladwa, ${ }^{*}$ M K Baig, K Singh, M Sayegh. Department of General and Laparoscopic Colorectal Surgery, Worthing Hospital, Worthing, UK

Introduction To systematically analyse the randomised trials comparing single incision laparoscopic cholecystectomy (SILC) vs conventional laparoscopic cholecystectomy (CLC).

Methods The meta-analysis was conducted according to the Quality of Reporting of Meta-analysis (QUORUM) standards. Standard electronic databases were searched to retrieve relevant randomised trials comparing SILC vs CLC and analysed systematically using RevMan ${ }^{\circledR}$. Summated outcomes were expressed as RR and standardised mean difference (SMD).

Results Eleven randomised, controlled trials encompassing 859 patients were retrieved for the electronic databases. In the random effects model, post-operative pain, post-operative complications, length of hospital stay, cosmesis score, conversion rate, additional ports insertion rate and time for return to normal activities were statistically comparable between two techniques of cholecystectomy. The SILC is associated with longer operative time. However, there was significant heterogeneity among included trials. Conclusion SILC does not offer any significant advantage over CLC to treat benign gallbladder disorders. CLC may assiduously be used for this purpose.

Competing interests None declared.

\section{PWE-135 RETROSPECTIVE AUDIT OF MANAGEMENT OF PATIENTS ADMITTED TO INTENSIVE CARE UNIT (ITU) WITH SEVERE ACUTE PANCREATITIS (SAP)}

doi:10.1136/gutjnl-2012-302514d.135

0 Jalil, ${ }^{*}$ C Patel, A Kambal, A Iqbal, A Rasheed. Department of General Surgery, Royal Gwent Hospital, Newport, UK

Introduction Atlantaclassification stratifies acute pancreatitis (AP) into mild and severe. Severe acute pancreatitis (SAP) is best managed in HDU or ITU setting and associated with high mortality and morbidity despite best efforts at attaining early diagnosis and timely intervention.

Aim To compare management strategies and mortality of patients admitted to ITU with SAP against national standards and study the group who succumbed to their disease in detail in an attempt to define the circumstances that lead to this event and identify the most accurate prognostic indicators in this group of patients.

Methods Retrospective audit of management and outcome of consecutive patients admitted to ITU with SAP during the period of 2007-2010. The development of necrosis, infected necrosis (IN) or organ failure $(\mathrm{OF})$ was recorded. Patients were classified into group I (No necrosis or OF), group II (sterile necrosis or transient OF), group III (IN or persistent OF) and group IV (infected necrosis and persistent $\mathrm{OF}$ ). The four groups were compared regarding the clinical course, radiological/surgical intervention, any post-intervention complications, use of antibiotics/antifungal and nutritional support. Results 51 patients were admitted to ITU with SAP (APACHE II $>8$, modified Glasgow score $>3$ ) during the period of 2007-2010. All cases fulfilled the Atlanta criteria of SAP. Median age: $66 \pm 17.5$. The pancreatitis was alcohol induced in $12 \%$ and due to gallstones in $59 \%$ of patients; no cause was found in $25 \%$ of patients. Median ITU stay was 3.23 days. The overall mortality rate during the study period (3 years) was 38\% ( $n-19)$ above national standard of $30 \%$. All seven patients in group IV died, five of them underwent necrosectomy and one had CT guided drainage of infected acute fluid collection. The Abstract PWE-135 table 1 shows the total number of patients and respective mortality of SAP in all four groups. Forty-one patients $(80 \%)$ received antibiotics and 35 patients $(69 \%)$ had nutritional support but neither of them seems to have a significant impact on survival ( $\mathrm{p}=0.6$ and 0.06 respectively). Outcome (death) correlated with organ dysfunction criteria (Atlanta criteria and APACHE II score).

Abstract PWE-135 Table 1 The mortaltiy of SAP in the different groups

\begin{tabular}{lccc}
\hline Group & Total number & Mortality & $\%$ of mortality \\
\hline I & 12 & 0 & $0 \%$ \\
II & 2 & 0 & $0 \%$ \\
III & 30 & 12 & $40 \%$ \\
IV & 7 & 7 & $100 \%$ \\
\hline
\end{tabular}

